\title{
Advancement in OPEFT Model
}

\author{
Vrushali V. Kelkar \\ Department of Electronics and \\ Telecommunications, \\ Goa College of Engineering \\ Ponda, Goa
}

\author{
Shrinivas S. Joshi \\ Department of Electronics and \\ Telecommunications, \\ Goa College of Engineering \\ Ponda, Goa
}

\author{
Rajesh B. Lohani \\ Department of Electronics and \\ Telecommunications, \\ Goa College of Engineering \\ Ponda, Goa
}

\begin{abstract}
High speed low cost monolithically integrated photo electronic circuits using metal semiconductor field effect transistors are highly using for variouswavelength optical communications. In recent years GaAs is more extensively used for the fabrication of ion implanted MESFET than any other material. This paper discusses the methods used to solve continuity equation and their comparison with respect to the voltage-current characteristics and the $\mathrm{AC}$ characteristics in dark and illuminated condition.
\end{abstract}

\section{Keywords}

Optoelectronic, photovoltage, OPFET, semi-transparent.

\section{INTRODUCTION}

As the name suggests optoelectronics is a branch of physics that mainly deals with the combination of opto i.e. light and electronics. Devices in which such interaction can suitably take place, usually accompanied by an energy conversion process (e.g. from electrical to optical vice-versa), are called optoelectronic devices.

Although semiconductors have been very useful for the development of microelectronics, they face some important drawbacks. The bandgap (i.e. the difference between top of valence band and bottom of conduction band) of silicon semiconductor is indirect (i.e. the photon is emitted only after the electron passes from valence band through an intermediate state to the conduction band). This implies that they emit light very poorly and their absorption coefficients are low. Therefore silicon cannot be considered as a universal semiconductor material since it cannot perform many important functions. Compound semiconductor materials composing of elements from two or more different groups of the periodic table provided many of the desired properties and could be synthesized without much difficulty. E.g III-V, IIIV, IV-IV, IV-VI compounds. Most commonly used are GaAs and InP.

The GaAs OPFET has received much attention due to its potential application as a high-speed optical detector. The optical terminal provides an extra terminal to the device without any decoupling structure [4].

OPFET may be used as a dual-gate memory device in computers with one gate being real (where voltage is applied) and the other gate is virtual (where radiation is allowed to fall). It can be used as a high-speed and high-power device and power handling capacity can be controlled by controlling the incident photon flux density [1].

\section{THEORY}

The schematic structure of OPFET is as shown in Figure.1

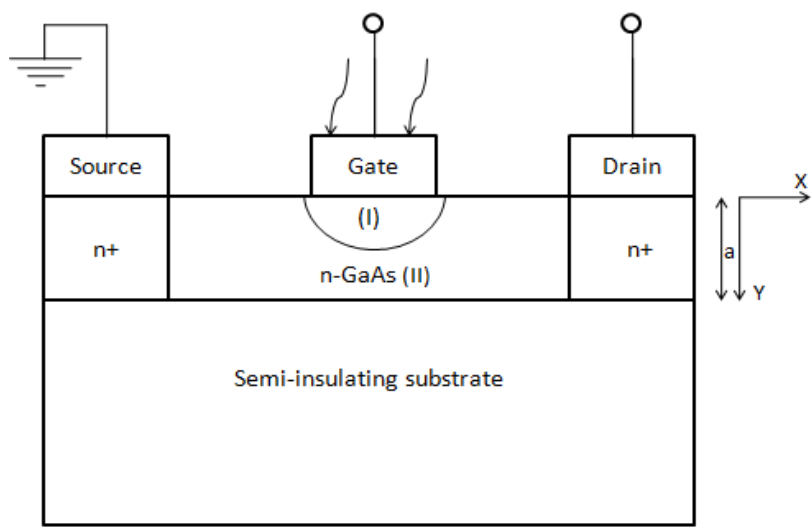

Fig 1: Cross-sectional view of an OPFET [2].

In Figure.1, The conventional way of illuminating and OPFET is by allowing the optical radiation to fall on the semi-transparent schottky gate of the device [11]. For making the gate semi-transparent the metal gate thickness should be less than $100 \dot{A}[5]$. The drain-source current flows along the $\mathrm{x}$-direction and the illumination is incident along the $\mathrm{y}$ direction on the semi-transparent gate of the device. Electronhole pairs are generated due to absorption of photons in the neutral channel region (II) and the schottky junction depletion region (I). The optically generated electrons move from source to drain in the channel region when a drain-source voltage is applied while the holes move towards the substrate. When these holes cross the junction, a photo-voltage is developed. This voltage being forward biased reduces the depletion width of the Schottky junction [3][6]. Under illumination the photo generated electrons and holes in the neutral and depletion regions are obtained by solving the $\mathrm{dc}$ continuity equations [9].

The schematic structure of the device is shown in Fig1. Assuming steady state condition, the spatial variation of photogeneration rate with space per unit volume is given by:

$$
G=\alpha \varphi e^{-\alpha y}
$$

where $\mathrm{G}$ is the generation rate per unit volume within the depletion region below the gate. $\Phi$ is the photon flux density per unit area per second, $\alpha$ is the photon absorption coefficient 
per unit length and $y$ is the direction perpendicular to the surface.

The ion-implanted region of the device is defined as a symmetrical Gaussian distribution function and expressed as:

$$
N_{D}(y)=\frac{Q}{2 \pi^{1 / 2} \sigma} \exp -\left(\frac{y-R p}{2 \sigma}\right)^{\frac{1}{2}}-N_{A}
$$

Where $\mathrm{N}_{\mathrm{D}}(\mathrm{y})$ is the substrate doping concentration per unit volume, $\mathrm{Q}$ is the implant dose per unit area, $\sigma$ and $\mathrm{Rp}$ are the implant straggle and range parameters in units of lengths.[2]

For low injection case, the flow of optically generated carriers is governed by the current continuity equations given by:

$$
\frac{\partial n(y, t)}{\partial t}=\frac{1}{q} \frac{\partial \operatorname{Jn}(y, t)}{\partial y}+G-\frac{n(y, t)}{\tau_{n}}
$$

for electrons and

$$
\frac{\partial p(y, t)}{\partial t}=\frac{1}{q} \frac{\partial J p(y, t)}{\partial y}+G-\frac{n(y, t)}{\tau_{p}}
$$

for holes,

where $\tau_{\mathrm{p}}$ is the minority carrier's life time and is assumed same for both holes and electrons.

The current equations consist of both drift and diffusion current and are given by:

$$
m(y, t)=q v_{y} n(y, t)+q D_{n} \frac{\partial n(y, t)}{\partial t}
$$

for electrons and

$$
I p(y, t)=q v_{y} p(y, t)+q D_{p} \frac{\partial p(y, t)}{\partial t}
$$

for holes, where $\mathrm{Jn}(\mathrm{y}, \mathrm{t})$ and $\mathrm{Jp}(\mathrm{y}, \mathrm{t})$ are the current densities due to photogenerated carriers along the y-direction, vy is the drift velocity in the y-direction and is assumed constant and independent of the field. Dn and Dp are the diffusion coefficients for electrons and holes respectively. The region can be divided into two regions: Region I-the depletion region below the Schottky gate; and Region II-the channel or bulk region.

\subsection{Calculation of photovoltage}

The photovoltage across the Schottky junction is calculated using the relation:

$$
V_{o p}=\frac{K T}{q} \ln \frac{J_{p}(0)}{J_{s}}
$$

Where is the current density of the number of holes crossing the surface of the device and is the minority carrier current density[7].

\subsection{Drain-source current}

The total channel charge is due to the ion implantation (Qion), the excess electrons in the depletion region (Qdep) and the excess electrons in the bulk (Qbulk) and is written as [3]:

$$
Q_{t}=Q_{\text {dep }}+Q_{\text {bulk }}+Q_{\text {ion }}
$$

The photocurrent is calculated using the electron continuity equation i.e equation (3).

The drain-source current is calculated using gradual channel approximation and is obtained using the relation:

$$
I_{D S}=\frac{q \mu z}{L} \int_{0}^{v d s} Q_{t} d v=I_{d e p}+I_{b u l k}+I_{\text {ion }}
$$

Where is the carrier mobility, $\mathrm{L}$ is the channel length, $\mathrm{z}$ is the channel width and $\mathrm{V}_{\mathrm{ds}}$ is the drain-source voltage[10].

\section{NUMERICAL TECHNIQUE}

The continuity equation for electrons has been solved analytically and numerically. This number of electrons has been used to calculate the amount of charge which is the integration or summation of the number of electrons in each space step. Then the amount of charge is calculated for two different time steps. The difference between the two divided by the time step gives the current [8].The numerical method used is the Leap Frog method.

\section{RESULTS AND DISCUSSIONS}

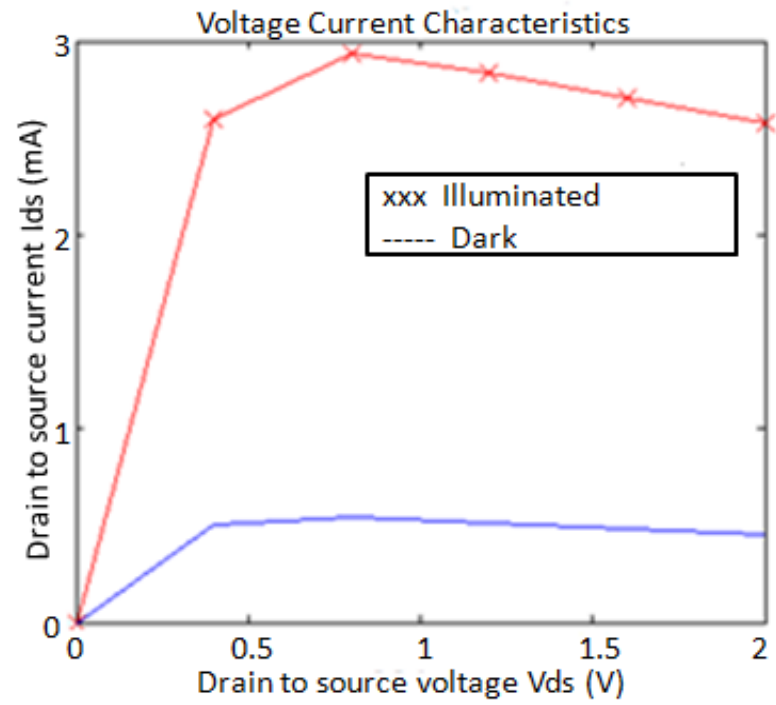

Figure 2: I-V characteristics of the device under dark and illuminated condition obtained using analytical method. 


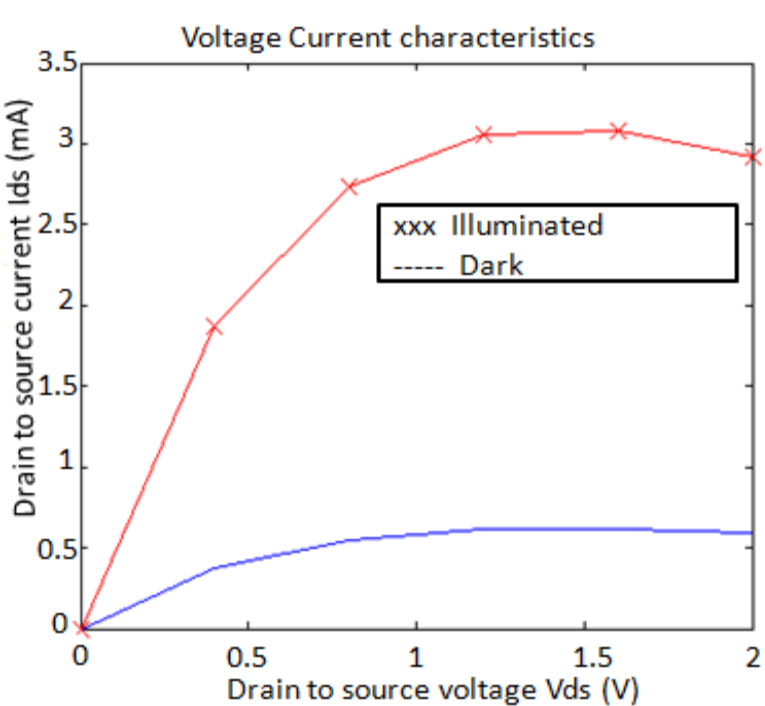

Figure 3: I-V characteristics of the device under dark and illuminated condition obtained using numerical method.

In the above figures it is observed that the current begins to increase with radiation flux density in the illuminated condition. This is because with the increase in the radiation flux density, the volume generation rate of carriers increases. Hence more carriers are generated at given time thus increasing the current. Also with the increase in the radiation flux density, the photovoltage generated increases and due to the photovoltaic effect there is the increase in the channel width which in turn increases the current.

From figure 2 and figure 3 it is observed that the I-V characteristics obtained using numerical method is gradually increasing to its saturation as compared to the I-V characteristics obtained using analytical method providing a better approximation.

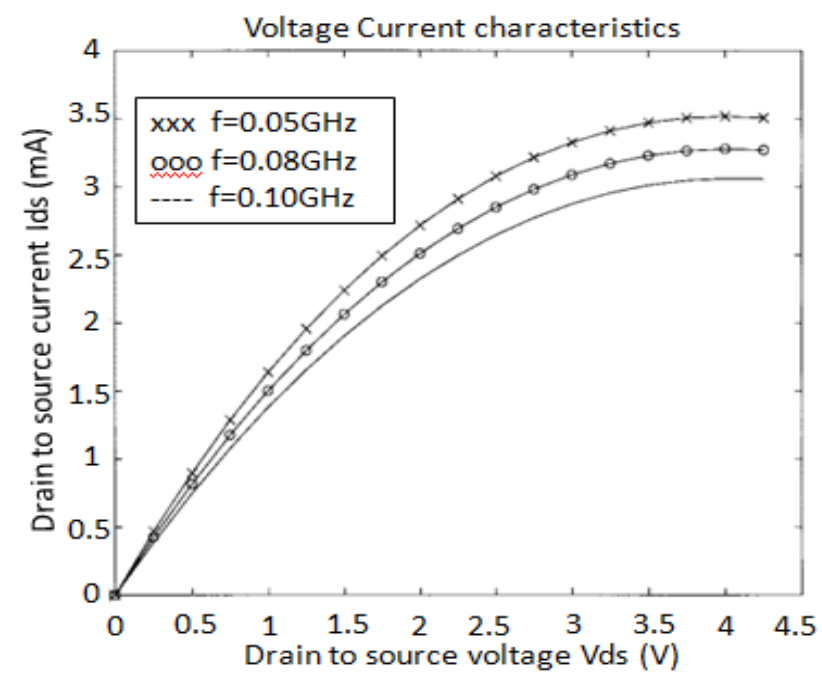

Figure 4: Plot of drain-source current versus drain-source voltage for different modulation frequencies solved using analytical method keeping the radiation flux density constant at $10^{23} \mathrm{~m}^{2} / \mathrm{s}$.

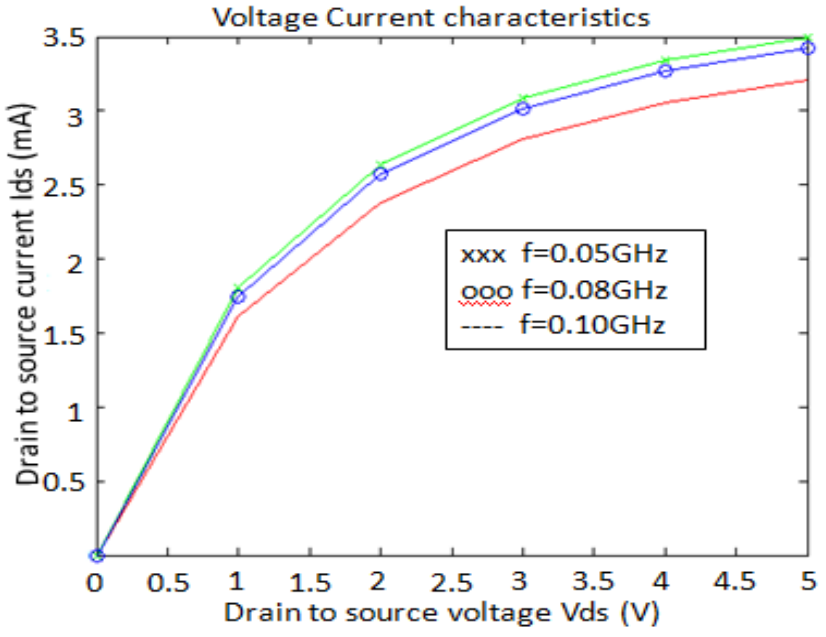

Figure 5: Plot of drain-source current versus drain-source voltage for different modulation frequencies solved using numerical method keeping the radiation flux density constant at $10^{23} \mathrm{~m}^{2} / \mathrm{s}$.

It is observed from figure 4 and figure 5 that the results obtained using analytical (experimental results) and numerical method are almost similar. Hence is can be stated that the solution obtained by numerical method is correct and verified.

\section{CONCLUSION}

The continuity equation for electrons has been solved using two different numerical method and the I-V characteristics of the front illuminated device for dark and illuminated condition have been plotted. The results obtained reveal that radiation flux density plays a very important role in the performance of highspeed devices. From the behavior of the I-V characteristics it is clear that the numerical Method is more convenient as compared to analytical method, as the I-V curve saturates gradually in the numerical Method. Also, it provides a better approximation as half time step is considered. It is a second order method which handles both space as well as time dependent equation simultaneously. It is simple. It requires less storage. It has global stability. Also, the device may be considered as a potential candidate for the design of high speed optical detector.

\section{ACKNOWLEDGMENTS}

Authors wish to thank Dr. B.B. Pal, ITBHU, Dr.R.P.R.CAiyar, CRNTS IITB, for providing constant help and necessary guidance and Mr. Vivek Kamat, DTE Goa for providing encouragement.

\section{REFERENCES}

[1] Nandita S. Mishra, V. K. Singh, and B. B. Pal, "Effects of radiation and surface recombination on the characteristics of an ion-implanted GaAs MESFET," IEEE Trans. Electron Devices, vol. 37, pp. 2-10,1990.

[2] P.Chakrabarti, N.L. Shreshtha, S.Srivastava and V. Khemka, "An improved model of ion-implanted GaAs OPFET", IEEE Trans. on Electron Devices Vol. 39, No.5, May 1992. 
[3] NanditaSaha Roy and B. B. Pal, "Frequency-Dependent OPFET Characteristics with Improved Absorption under Back Illumination", Journal of Lightwave Technology, Vol. 18, No. 4, April 2000..

[4] N.S.Roy, B.B.Pal and R.U. Khan, "Effect of substrate illumination on an ion impantedGaAs OPFET" IEE Proc.Optoelectron., Vol. 147, No. 3, June 2000

[5] Jaya V. Gaitonde and Rajesh B. Lohani, "One Dimensional Finite Difference Simulation of Back Illuminated OPFET", Proceedings of International Conference \& Workshop on Emerging Trends in Technology 2011, Volume-II, February 25th \& 26th 2011.

[6] Dr.B.K.Mishra, Lochan lolly and S.C.Patil, "InGaAs a next generation material for photodetector",Journal of Selected Areas in Microelectronics (JSAM), April Edition, 2011.
[7] Robert Soares, "GaAs MESFET Circuit Design."

[8] Vrushali V. Kelkar and Dr.R.B.Lohani, “OPFET characteristics considering the effect of modified active layer material", Proceedings of International Conference \& Workshop on Emerging Trends in Technology 2011, Volume-II, February 24th \& 25th 2012.

[9] S. M. Sze, Physics of Semiconductor Devices, 2nd ed. New York: Wiley, 1983, pp. 755

[10] Jaya T, Kannan V., "Analytical Model for I-V Characteristics of Buried Gate MESFET", International Journal of Advanced Science and Technology Vol.17, April 2010.

[11] Nandita Saha Roy,B.B. Pal and R.U.Khan,"Analysis ofGaAs OPFET with Improved Optical Absorption under Back Illumination," IEEE Transactions Election Devices, Vol.46, pp.2350-2353, Dec 1999 